\title{
Electromagnetic Problems Solving by Conformal Mapping: A Mathematical Operator for Optimization
}

\author{
Wesley Pacheco Calixto, ${ }^{1,2,3}$ Bernardo Alvarenga, ${ }^{1,3}$ \\ Jesus Carlos da Mota, ${ }^{4}$ Leonardo da Cunha Brito, ${ }^{1}$ \\ Marcel Wu, ${ }^{3}$ Aylton José Alves, ${ }^{3}$ Luciano Martins Neto, ${ }^{3}$ \\ and Carlos F. R. Lemos Antunes ${ }^{2,3}$ \\ ${ }^{1}$ Electrical \& Computer Engineering School, Federal University of Goias (UFG), \\ Avenda Universitaria, 1488 Qd. 86 Bl., 74605-010 Goiania, GO, Brazil \\ ${ }^{2}$ Electrical Engineering and Computers Department of the Faculty of Sciences and Technology, \\ University of Coimbra, 3030-290 Coimbra, Portugal \\ ${ }^{3}$ Electromagnetism and Electric Grounding Systems Nucleus Research and Development, \\ Department Electrical Engineering, Federal University of Uberlandia, 38400-902 Uberlandia, MG, Brazil \\ ${ }^{4}$ Institute of Mathematics $\mathcal{E}$ Statistics (IME), Federal University of Goias, Campus II, \\ 74001-970 Goiania, GO, Brazil
}

Correspondence should be addressed to Wesley Pacheco Calixto,wpcalixto@ieee.org

Received 22 September 2010; Accepted 10 December 2010

Academic Editor: Piermarco Cannarsa

Copyright (C) 2010 Wesley Pacheco Calixto et al. This is an open access article distributed under the Creative Commons Attribution License, which permits unrestricted use, distribution, and reproduction in any medium, provided the original work is properly cited.

\begin{abstract}
Having the property to modify only the geometry of a polygonal structure, preserving its physical magnitudes, the Conformal Mapping is an exceptional tool to solve electromagnetism problems with known boundary conditions. This work aims to introduce a new developed mathematical operator, based on polynomial extrapolation. This operator has the capacity to accelerate an optimization method applied in conformal mappings, to determinate the equipotential lines, the field lines, the capacitance, and the permeance of some polygonal geometry electrical devices with an inner dielectric of permittivity $\varepsilon$. The results obtained in this work are compared with other simulations performed by the software of finite elements method, Flux 2D.
\end{abstract}

\section{Introduction}

The conformal mapping simplifies some solving processes of problems, mapping complex polygonal geometries and transforming them into simple geometries, easily to be studied. This transformations became possible, due to the conformal mapping property to modify only the polygon geometry, preserving the physical magnitudes in each point of it [1]. 
In this work, the selected problems have only continuous second-order derivatives, $u$ and $v$ with respect to $x$ and $y$ in a region of the complex plane $\mathrm{C}$.

Under these conditions, the real and the imaginary parts of an analytical function satisfies the Laplace equations, that is, functions such as $u(x, y)$ and $v(x, y)$. These are known as harmonic functions [2].

All the electrical devices, work based on the action of electrical fields produced by electrical charges, and magnetic fields produced by electrical currents. To understand the working principle of these electrical devices, its fields lines must be evaluated inside and around then, allowing a spatial visualization of the phenomena [3].

In another words, field mapping must be produced, describing the behavior of the electric and magnetic phenomena. These maps typically represents flux and fields lines, equipotential surfaces and densities distributions, having information about field intensity, potential difference, energy storage, charges, current densities, and so forth. Getting the field mapping is possible by solving the Laplace equation. However, these differential equations are rather complex solution, and in most practical cases, only have a numerical solution.

Some works have been produced, using optimized processes applied in conformal mappings, intending to simplify certain electromagnetic problems [4]. This paper aims to show that some difficult electromagnetic problems can be easily solved, using simple computational and mathematical tools.

For a better comprehension of the process, the analytical calculation of the direct Schwarz-Christoffel Transformation is defined in Section 2. Section 3 presented the calculation of the inverse Schwarz-Christoffel Transformation, within the employment of an elliptic integral of first kind, whose inverse is known as the Jacobi function. In Section 4 the Genetic Algorithm employed in this work is described and a new mathematical operator is developed, introducing a new method to be used in optimization techniques. In Section 5 the proposed methodology to solve electromagnetic problems is described and in Section 6 the results are exposed.

\section{Direct Schwarz-Christoffel Transformation}

The Schwarz-Christoffel Transformation is a conformal mapping of the complex plane C in $\mathrm{C}$ that maps the real axis onto the boundary of a polygon and the upper half plane of the complex plane into the interior of this polygon [1].

Consider a polygon in $\mathrm{C}$, of $N$ sides, with its vertices in $w_{1}, w_{2}, \ldots, w_{N}$, ordered in the counterclockwise, with corresponding internal angles denoted by $\beta_{1}, \beta_{2}, \ldots, \beta_{N}$ and external angles denoted by $\pi \cdot \alpha_{n}, n=1,2, \ldots, N$. The Schwarz-Christoffel Transformation in the integral form is defined by $[2,5]$

$$
w=f(z)=A+C \int \prod_{n=1}^{N} \frac{1}{\left(z-x_{n}\right)^{\alpha_{n}}} d z,
$$

where $A$ and $C$ are complex constants. The points $x_{1}, x_{2}, \ldots, x_{N}$ over the real axis, called prevertices, are mapped into the vertices $w_{1}, w_{2}, \ldots, w_{N}$. It is convenient to assume that $z_{0}=$ $f^{-1}\left(w_{0}\right)=\infty$, because if infinity is not a prevertex, its image will be a new vertex with the corresponding internal angle equal to $\pi[6]$.

The complex constants $A$ and $C$ and the prevertices $x_{1}, x_{2}, \ldots, x_{N}$, are referred as parameters of the Schwarz-Christoffel Transformation. 
Figure 1 illustrates an example of the Schwarz-Christoffel Transformation for an eightside polygon. The segments $\overline{x_{i} x_{i+1}}, i=1,2, \ldots, 7$, on the real axis of the complex plane, are mapped, respectively, into the sides $\overline{w_{i} w_{i+1}}$ of the polygon. The semi-interval $\left(-\infty, x_{1}\right]$ is mapped into the segment $\overline{w_{0} w_{1}}$, and the semi-interval $\left[x_{8},+\infty\right)$ is mapped into the segment $\overline{w_{8} w_{0}}$.

The Schwarz-Christoffel Transformation is a conformal mapping of the half-plane $y \geq$ 0 , except in critical points $x_{n}$. Notice that $f$ is not continuous at points $x_{n}$. Once, when $z$ move on the real axis, from left to right of $x_{n}$, the argument of the number $z-x_{n}$ changes from $\pi$ to $z e r o$, the other arguments of $z-x_{i}, i \neq n$, do not change, while the argument of $f(z)-f\left(x_{n}\right)$ changes from $\pi \cdot \alpha_{n-1}$ to $\pi \cdot \alpha_{n}[7,8]$, see Figure 1 .

Given a polygon with $N$ sides, as Figure 1, where the vertices $w_{1}, w_{2}, \ldots, w_{N}$, and the parameters $\alpha_{n}$ that define the external angles are known. Therefore, utilizing (2.1) with $A=0$ and $C=1$, the prevertices $x_{1}, x_{2}, \ldots, x_{N}$ can be determined by the following integrals (2.2)

$$
\begin{gathered}
w_{1}=\int_{-\infty}^{x_{1}} \prod_{n=1}^{N}\left(z-x_{n}\right)^{-\alpha_{n}} d z, \\
w_{2}=w_{1}+\int_{x_{1}}^{x_{2}} \prod_{n=1}^{N}\left(z-x_{n}\right)^{-\alpha_{n}} d z, \\
\vdots \\
w_{N}=w_{N-1}+\int_{N-1}^{x_{N}} \prod_{n=1}^{N}\left(z-x_{n}\right)^{-\alpha_{n}} d z .
\end{gathered}
$$

Now, considering a polygon with $N$ sides and vertices $w_{1}, w_{2}, \ldots, w_{N}$. One of the goals of this work is to find the corresponding prevertices, $x_{1}, x_{2}, \ldots, x_{N}$, utilizing a Genetic Algorithm.

\section{Calculation of the Inverse Schwarz-Christoffel Transformation}

The calculation of the inverse Schwarz-Christoffel Transformation is mathematically known as an inverse problem. The major part of the inverse problems are solved by nonlinear optimization techniques.

There are several numerical methods to estimate the parameters of the Inverse Schwarz-Christoffel Transformation. In [9], Trefethen uses a method based on equations obtained from relations between the polygon vertices. He proposes a reverse way utilising the Newton Method, having a practical difficulty to establish the first point or parameter $x_{1}$, which initiates the algorithm. After, differential equations are utilized, which must be integrated between consecutive prevertices points $x_{1}, x_{2}, \ldots, x_{N}$, producing numerical inconsistency in some cases.

Driscoll and Vavasis in [10], proposes a method based in Cross-Ratios and Delaunay triangulation. Costamagna in [11], introduces an algorithm based on optimization techniques. For few vertices polygons, this last method, estimates the parameters with a satisfactory accuracy. Driscoll and Trefethen in [12] presents a method for the inversion of 


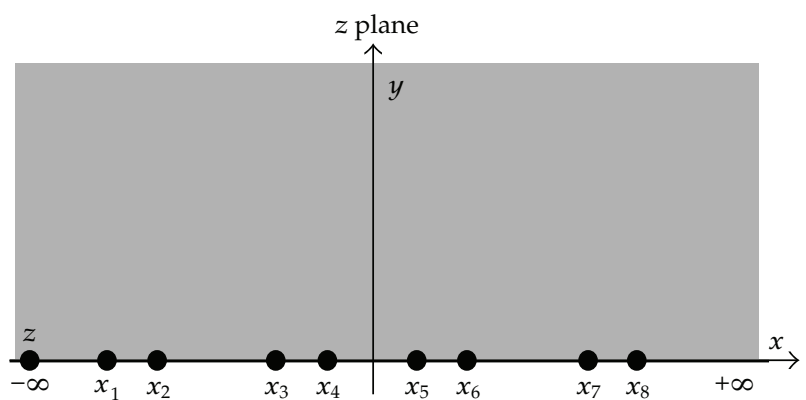

(a)

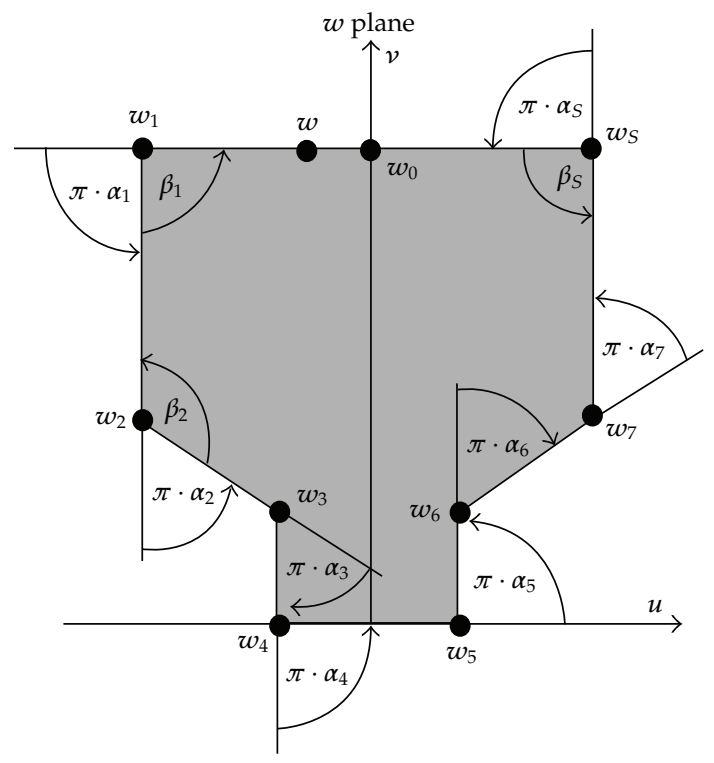

(b)

Figure 1: Mapping of a polygon through the direct Schwarz-Christoffel transformation.

the Schwarz-Christoffel Transformation, based on algebraic computation, which maps the polygon onto a disc, an infinite strip or a rectangle.

This section describes the analytical solution of the inverse Schwarz-Christoffel Transformation for the rectangle Figure 2. In this case, the Schwarz-Christoffel Transformation is given by

$$
w(z)=\int_{z_{0}}^{z} \frac{d z}{\left(z-x_{1}\right)^{1 / 2}\left(z-x_{2}\right)^{1 / 2}\left(z-x_{3}\right)^{1 / 2}\left(z-x_{4}\right)^{1 / 2}} .
$$

Choosing the imaginary axis as the symmetry axis of the rectangle and as prevertices of the vertices, the points $x_{1}=-a, x_{2}=-1, x_{3}=1$, and $x_{4}=a$, with $a>1$, the integral (3.1) can be written in the following form [13]:

$$
w(z)=\int_{z_{0}}^{z} \frac{d z}{\sqrt{\left(z^{2}-a^{2}\right)\left(z^{2}-1\right)}}
$$




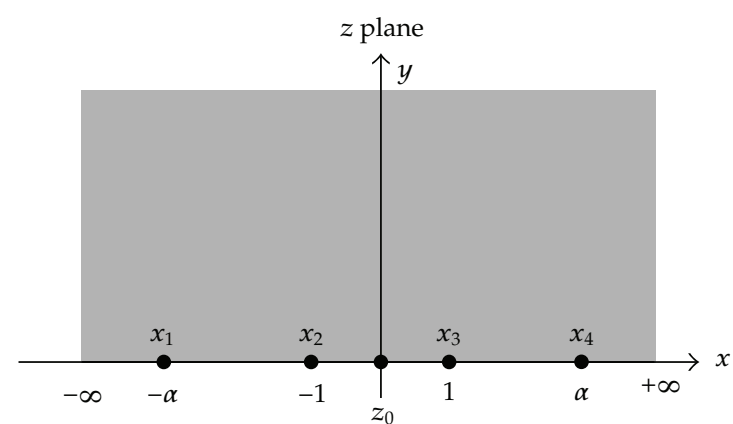

(a)

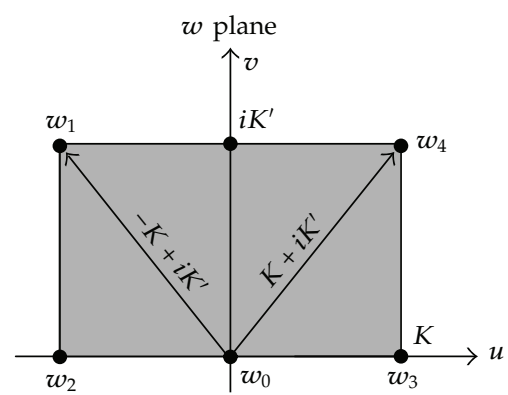

(b)

Figure 2: Schwarz-Christoffel transformation for the rectangle.

Making $z_{0}=0$ and $k=1 / a$, the integral (3.2) is transformed in:

$$
w(z)=\int_{0}^{z} \frac{d z}{\sqrt{\left(1-z^{2}\right)\left(1-k^{2} z^{2}\right)}}
$$

which is called an elliptic integral of first kind [14], and $k$ is the modulus of the elliptic integral with $0<k<1$. The $k^{\prime}=\sqrt{1-k^{2}}$ value is called elliptic integral building block.

The inverse mapping of (3.3) is known as the Jacobi elliptic function, denoted by:

$$
z(w)=\operatorname{sn}(w, k)
$$

where sn denotes the elliptic sine of $w$ for each fixed $k$.

Two important numbers in the elliptic integrals theory are known as the quarters of real and imaginary periods [14], respectively, defined by,

$$
\begin{gathered}
K=K(k)=\int_{0}^{1} \frac{d z}{\sqrt{\left(1-z^{2}\right)\left(1-k^{2} z^{2}\right)}}, \\
i K^{\prime}=i K\left(k^{\prime}\right)=i \int_{0}^{1} \frac{d z}{\sqrt{\left(1-z^{2}\right)\left(1-k^{\prime 2} z^{2}\right)}},
\end{gathered}
$$

One can observe that $K=w_{3}$ and $K+i K^{\prime}=w_{4}$, where $w_{3}$ and $w_{4}$ are the rectangle vertices of Figure 2.

In this case, the upper half-plane $y \geq 0$ is transformed by (3.3) onto the rectangle with vertices $-K+i K^{\prime},-K, K$, and $K+i K^{\prime}$, which corresponding prevertices are $-a,-1,1$, and $a$, as shown in Figure 2. The values of $k, k^{\prime}, K$, and $K^{\prime}$ can be calculated in function of $a$, since point $a$ is fixed on the real axis. Therefore, the calculation of the prevertices for the rectangle axis is made directly, simply fixing on a single point on the real axis.

Until today, there is no analytical formulation to calculate the parameters of the Inverse Schwarz-Christoffel Transformation, for polygons with more than four vertices. In the case of polygons with more than four vertices, numerical methods are used. In Section 1 some of 


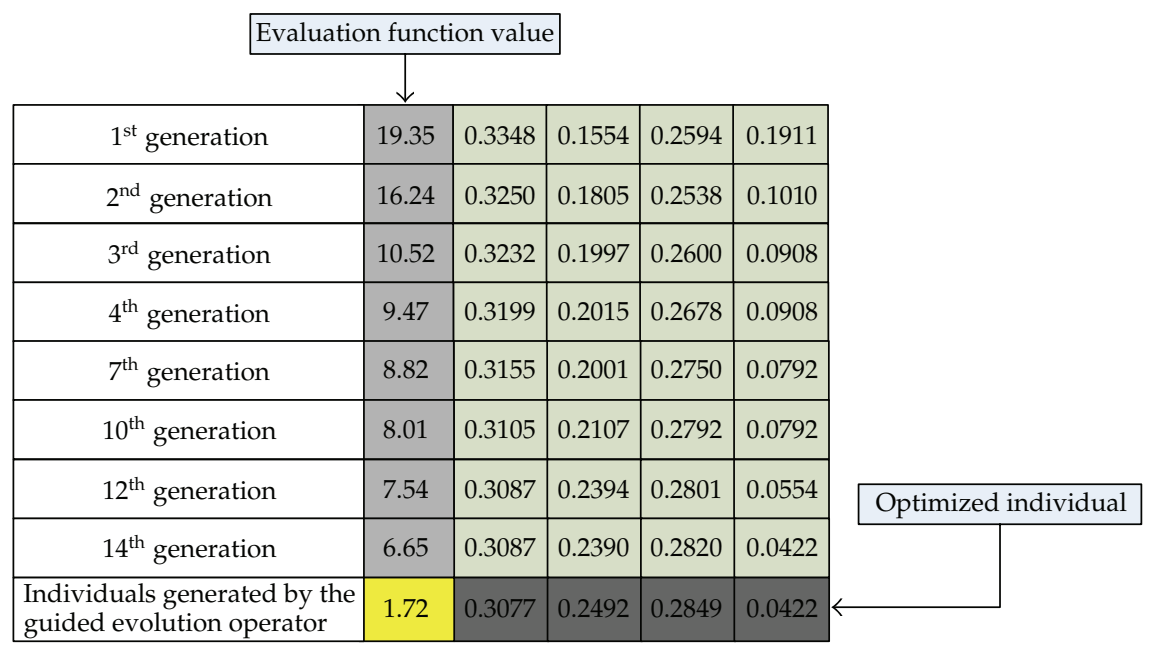

Figure 3: Guided Evolution operator.

these methods are cited. In this work, a Genetic Algorithm is utilized [15], to perform the parameters calculation.

\section{Genetic Algorithm}

Amongst the four paragons of the Evolutionary Computing, the Genetic Algorithm owns main position, once they constitute the most complete paradigm, gathering naturally all the fundamental ideas of evolutionary computing [15]. Genetic algorithms are stochastic methods with random search of optimal solutions. In the method, a population of individuals is maintained (chromosomes) representing possible solutions, being this population subjected to certain transformations (mutations and crossover), generating new and better candidates, which tend to improve the performance of the algorithm towards an optimal point or some optimized points [16].

In the genetic algorithm structure, the following operators are employed: Directed Crossover operator [15], Tournament Selection operator [17], Elitism [18], Crossover Operator with Multiple Descendants [19], Variable Mutation operator [17] and Guided Evolution operator.

All the operators mentioned in this work were the subject of several studies already published. However, the Guided Evolution operator was developed to be applied with the conformal mapping used in this work. The Guided Evolution operator saves only the best individual of each generation $g$. After a certain amount of generations $g+1$, there is a pattern to the aggregate value of each gene, as illustrated in Figure 3.

Using the values of the gene from the best individuals of each generation $g+1$, that is, column of 3rd at 6th of Figure 3, is made an polynomial extrapolation of these values with the value of the evaluation function (fitness), 2nd column Figure 3. Thus, the value found for each gene, forms a new individual with high fitness to the next generation. To produce the extrapolation, it is necessary to randomly choose a value for the evaluation function, lower than the current value and greater than the value determined in the genetic algorithm stopping criteria, in Figure 3, this value is illustrated in the last line, second column (1.72). 


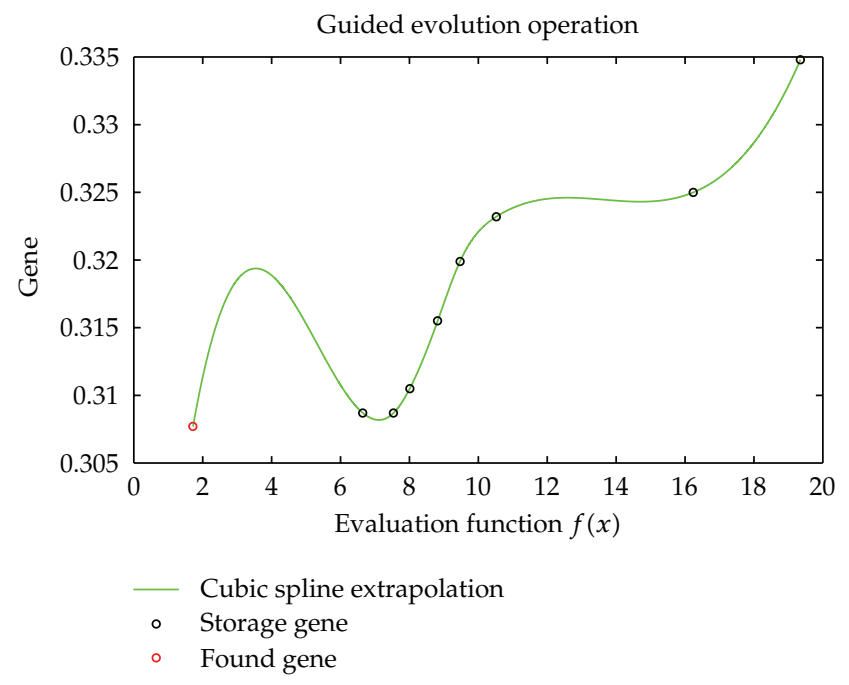

Figure 4: Extrapolation example for one column of genes.

The current value is 6.65 and the stopping criteria is $f(W)-1=0$, where $f(W)$ is defined as the evaluation function of the genetic algorithm, given by the following:

$$
f(W)=\frac{1}{N} \sum_{n=1}^{N} \sqrt{\frac{\left(u_{n+1}-u_{n}\right)^{2}+\left(v_{n+1}-v_{n}\right)^{2}}{\left(u_{n+1}^{\prime}-u_{n}^{\prime}\right)^{2}+\left(v_{n+1}^{\prime}-v_{n}^{\prime}\right)^{2}}},
$$

where $W=\left(w_{1}^{\prime}, w_{2}^{\prime}, \ldots, w_{N}^{\prime}\right)$. Function $f(W)$ defines a metric that allows comparison between the vertices of the polygon produced by the genetic algorithm, and the vertices of the expected polygon. The expected optimal value is $f(W)=1$.

In the production of the extrapolation, the Guided Evolution operator, considers the column of the evaluation function as the $x$-axis, and the column of genes, as the ordinate axis. This operator has the ability to extract the intrinsic characteristic of each column of genes and effectively insert all these features into a new individual. It is important to check the random value found (new value of the evaluation function) to be extrapolated, because if this value is far from the value of the current evaluation function and far near to the optimal value, will produce a superindividual.

There are several methods of extrapolation in the literature. The developed Guided Evolution operator can work with any of these methods, however, the operator will have best performance, according to the extrapolation method used, which should vary with the problem to be solved. In this work the cubic spline extrapolation was employed. This extrapolation is illustrated in Figure 4, where the plotted values of the evaluation function and the genes values were extracted from Figure 3.

\section{Proposed Methodology}

It is proposed to perform the calculation of some electromagnetical characteristics of polygonal geometry, in order to simplifying the study region. For this, the geometry of the 


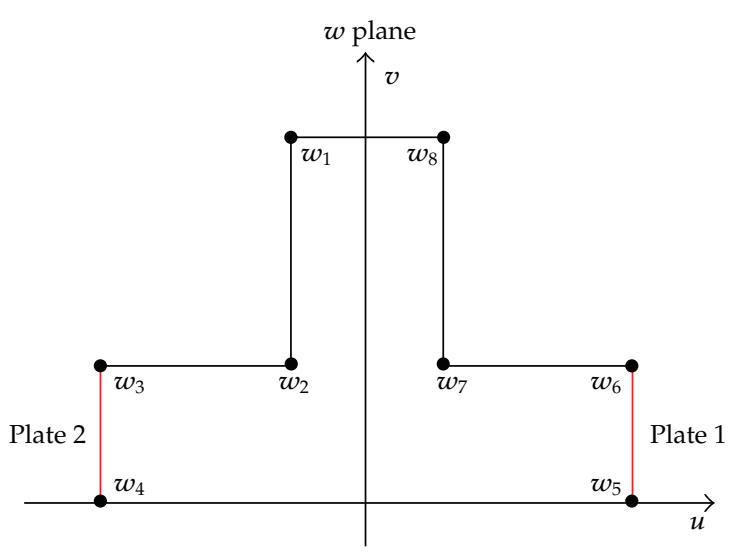

(a)

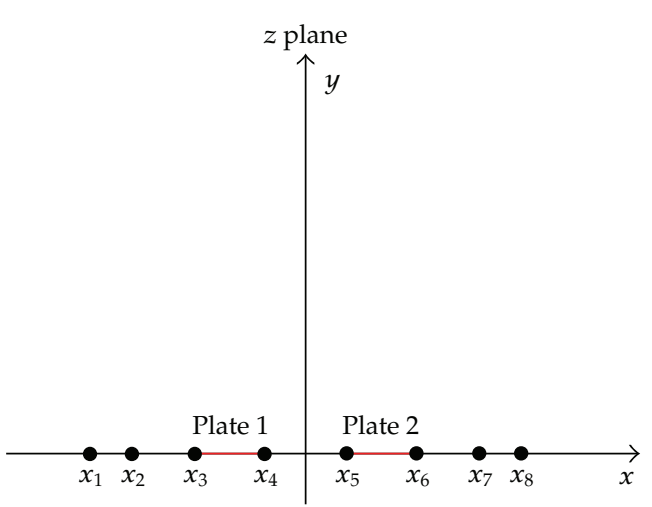

(b)

Figure 5: Inverse Schwarz-Christoffel transformation.

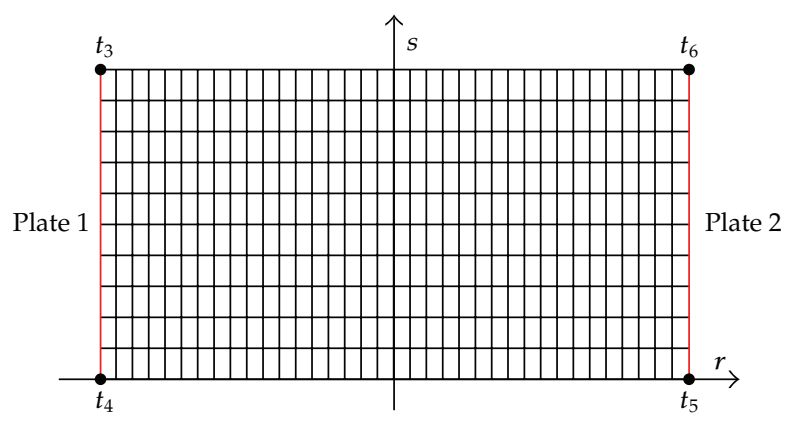

Figure 6: Equipotential and field lines in the auxiliary complex plane, $t$ plane.

studied device is transformed into a rectangle, using the Schwarz-Christoffel Transformation (2.1). Therefore, to study, for instance, a geometry as presented in Figure 5(a), it is firstly necessary to map the vertices of the complex $w$ plane into the prevertices of the complex $z$ plane, using the genetic algorithm for this, Figure 5(b).

Figure 5(a), regards two plates with distinct potentials: plate $\overline{w_{3} w_{4}}$, has a zero potential, and plate $\overline{w_{5} w_{6}}$, has a positive potential $V$. These plates are mapped, respectively, into the plates $\overline{x_{3} x_{4}}$ and $\overline{x_{5} x_{6}}$ of the complex $z$ plane [15], as illustrated in Figure 5(b). Utilizing the direct Schwarz-Christoffel transformation, the two plates of the complex $z$ plane, are mapped into a rectangle on the complex $t$ plane, denoted auxiliary complex $t$ plane. In this rectangle, one can perform the necessary calculations to solve electromagnetic problems and easily map the field and equipotential lines, as illustrated in Figure 6.

Using the Jacobi Elliptic Function (3.4), it is possible to map the rectangle traced lines at the complex $t$ plane, into the upper half-plane of the complex $z$ plane, Figure 7 and, using the parameters obtained by the genetic algorithm, one can map them into the complex $w$ plane, forming the original geometry of the study, as presented in Section 6. 


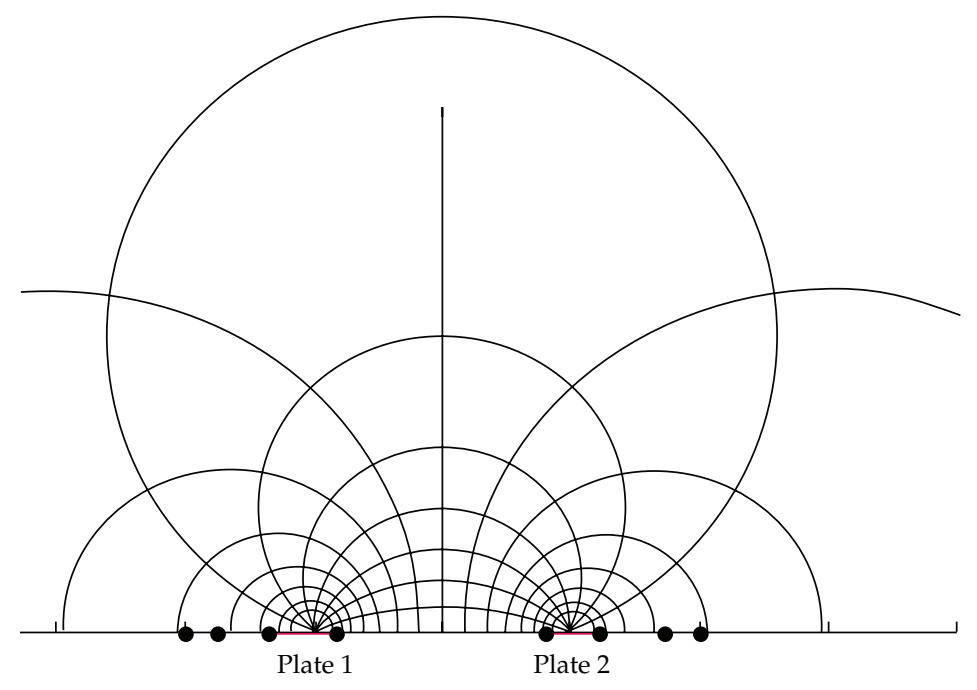

Figure 7: Equipotential and field lines in the complex $z$ plane.

\section{Results}

In this section the described method is applied in the calculation of the prevertices from the given vertices of some polygons with different geometries. As an application example, the Schwarz-Christoffel Transformation is used to calculate the equipotential lines and field lines in the air gap of an electrical machine. Also as an example, the capacitance of four devices are obtained and finally, the method is applied to calculate the permeance value of a device with cross-section and polygonal geometry. The results obtained of the capacitance and the permeance are compared with the results obtained from the finite element method (FEM), using the Flux 2D software [20].

\subsection{Field and Magnetic Equipotential Lines}

Using the methodology proposed in Section 5, Figure 8 illustrates two examples of field or flux lines and equipotential or induction lines in the air gap of an electric machine with a doubly slotted surface air gap. The mapping obtained by the proposed methodology (described at Section 6.1), illustrates flux lines penetrating the bottom of the slot, see Figure 8. Considering the rotor's surface at a null potential, and the stator's surface at a positive potential $V$, it is observed that the magnetic equipotential lines near the rotor surface (top) do not suffer influence from the opposite surface (stator's surface), thus as the magnetic equipotential lines near the stator surface (top) do not suffer influence from the rotor surface. The same is not true with the air gap induction middle line, which is influenced by both surfaces.This observation has great relevance in electrical machine designs, for the calculation of the correction coefficient of the slot opening [4].

\subsection{Capacitance of a Semicylindrical Plates Capacitor}

Capacitance is the property that a capacitive element presents of energy storage, in potential energy form, by means of electric fields. The capacitors have several applications in addition 

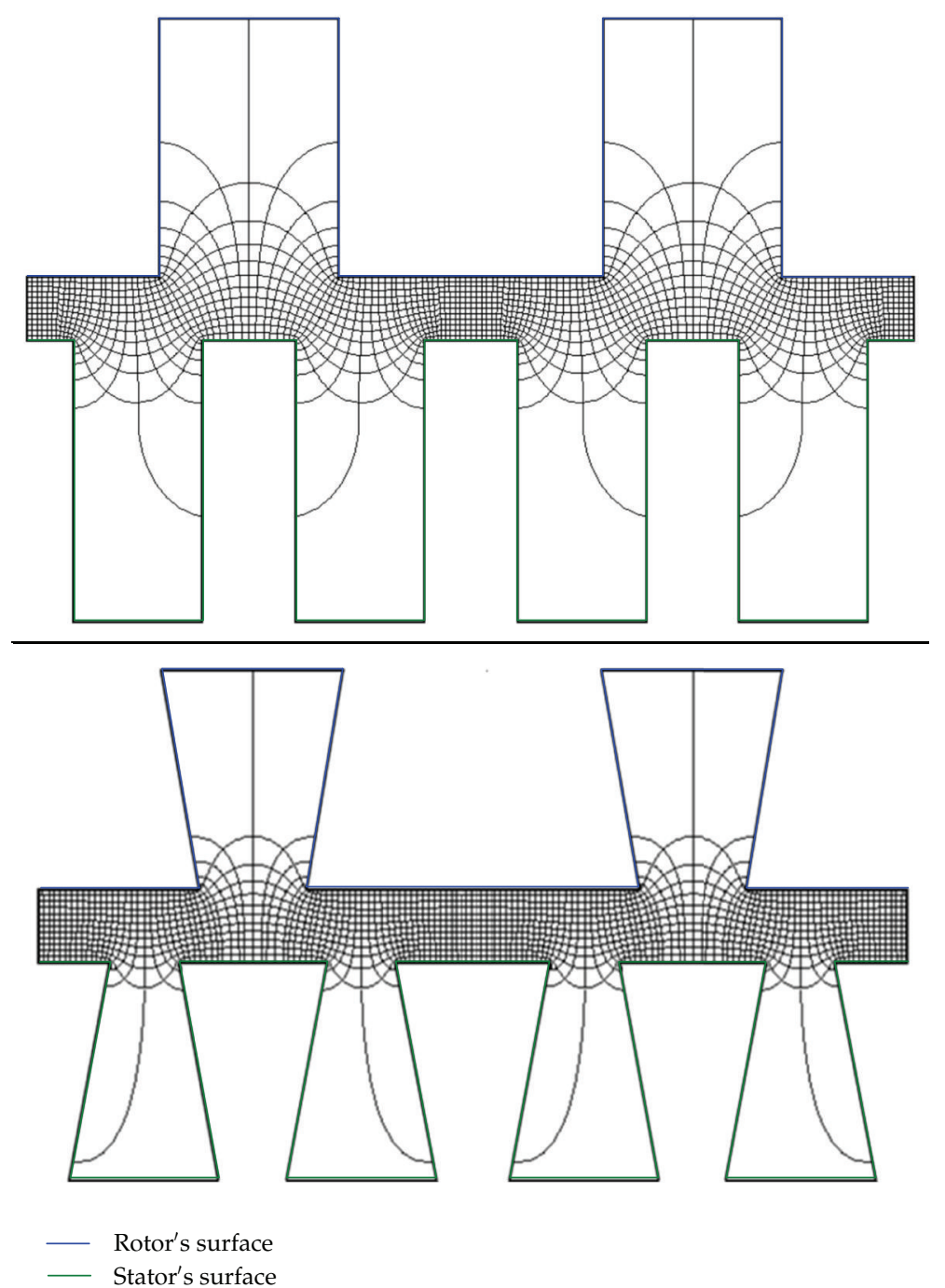

Figure 8: Flux and induction lines in the complex $w$ plane.

to serving as energy storage. They are important components in electrical circuits and are presented in a variety of sizes and shapes. However, the basic components of any capacitor is two insulated conductors of arbitrary shapes, with a dielectric material between them. The conductors are called as plates, regardless the geometry that they have. Parallel plates capacitors are very commonplace, hence, the need to find a rectangular geometry to facilitate the calculations.

For instance, a problem to determine the capacitance of a semicylindrical plates capacitor is illustrated at Figure 9, [21].

It is considered that Plate 1 has a positive potential $V$ and that Plate 2 has null potential. Inside the plates, there is a dielectric material of permittivity $\varepsilon(\mathrm{Fm}-1)$. It is also considered that the capacitor has depth $d(\mathrm{~m})$ and is immersed in a place of infinite permittivity. Assuming that the cross-section of the capacitor constitutes a polygon in the 
Plate 1

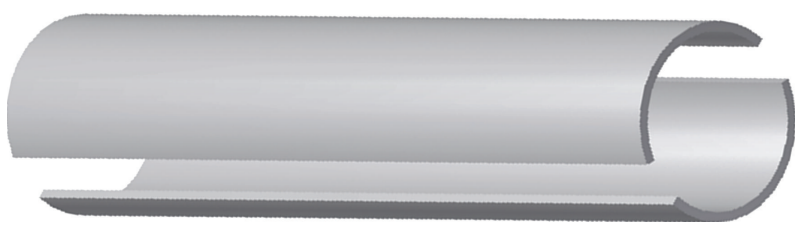

Plate 2

Figure 9: Capacitor of semicylindrical plates.

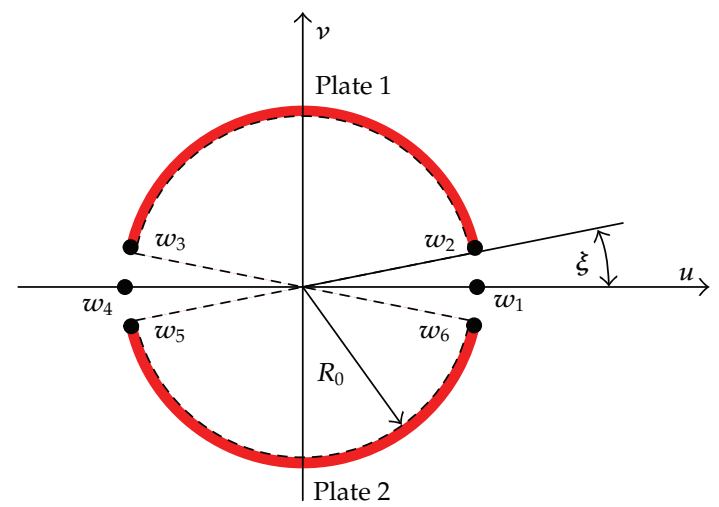

Figure 10: Points in the complex $w$ plane semicylindrical plates capacitor.

complex $w$ plane, the bilinear transformation $w(z)$, which maps the points of the complex $w$ plane into the points of the complex $z$ plane, is defined by

$$
w(z)=v \cdot \frac{w-R_{0}}{w+R_{0}}
$$

where $R_{0}(\mathrm{~m})$ is the radius of the capacitor and $\vartheta$ is a complex constant defined by

$$
\vartheta=\frac{e^{i \cdot \xi}+1}{e^{i \cdot \xi}-1}
$$

in which $\xi$ (rad) is associated to the angular spacing between the plates. Figure 10 illustrates the values $R_{0}, \xi$ and some relevant points to the transformation.

Relation (6.1) maps the points inside the circle defined by the capacitor, into points in the upper half-plane of the complex $z$ plane. Particularly, the points $w_{1}$ to $w_{6}$ on the circle of radius $R_{0}$ in the complex $w$ plane, are mapped into points $z_{1}$ to $z_{6}$ on the $x$-axis of the complex $z$ plane. Table 1 shows the correspondence between the points of $w$ and $z$ planes in general terms.

Adopting $R_{0}=5(\mathrm{~mm})$ and $\xi=\pi / 12$, the correspondence of the relevant points in $w$, $z$, and $t$ planes can be obtained, according to Table 2 .

To find the capacitance, an analogy with the method of mapping curvilinear squares of graph [3] is made. The division in the field $t$, must be performed, so that the subdivisions 
Table 1: Relationship between the $w$ and $z$ planes for the capacitor of semicylindrical plates.

\begin{tabular}{lc}
\hline$w$ plane & $z$ plane \\
\hline$w_{1}=R_{0}$ & $z_{1}=0$ \\
$w_{2}=R_{0} e^{i \cdot \alpha}$ & $z_{2}=1$ \\
$w_{3}=-R_{0} e^{-i \cdot \alpha}$ & $z_{3}=-\beta^{2}$ \\
$w_{4}=-R_{0}$ & $z_{4}=\infty$ \\
$w_{5}=-R_{0} e^{i \cdot \alpha}$ & $z_{5}=\beta^{2}$ \\
$w_{6}=R_{0} e^{-i \cdot \alpha}$ & $z_{6}=-1$ \\
\hline
\end{tabular}

Table 2: Relationship between the $w, z$, and $t$ planes for the semicylindrical plates capacitor.

\begin{tabular}{lcc}
\hline$w$ plane & $z$ plane & $t$ plane \\
\hline$w_{2}=4.82+1.29 i$ & $z_{2}=1.000$ & $t_{2}=1.57+0.00 i$ \\
$w_{3}=-4.82+1.29 i$ & $z_{3}=57.69$ & $t_{3}=1.57+5.44 i$ \\
$w_{5}=-4.82-1.29 i$ & $z_{5}=-57.69$ & $t_{5}=-1.57+5.44 i$ \\
$w_{6}=4.82-1.29 i$ & $z_{6}=-1.000$ & $t_{6}=-1.57+0.00 i$ \\
\hline
\end{tabular}

of the geometry becomes as close as possible of squares. The squares formed in the auxiliary complex $t$ plane, represent field cells. The mapping of squares or field cells of the $t$ plane in the complex $z$ plane produces the so-called curvilinear squares, see Figure 11 an illustration of curvilinear squares.

The theory associated with the curvilinear squares establishes that the device capacitance is equivalent to the capacitance of each cell, multiplied by the number of cells in parallel, divided by the number of cells in series. The accuracy of the method improves, according to the increasing of the domain subdivision $t$ or $z$, that is, with the increasing in the number of curvilinear squares. In the limit, the ratio between the number of cells in parallel and the number of cells in series is the ratio between the height of the plates and the distance between them in the complex $t$ plane. Thus, the capacitance of the device is given by

$$
C=\varepsilon \cdot \frac{K^{\prime} \cdot d}{2 \cdot K}
$$

where $K^{\prime}=t_{6}-t_{5}(\mathrm{~m})$ and $(2 \cdot K)=t_{5}-t_{3}(\mathrm{~m})$. As already mentioned, $\varepsilon\left(\mathrm{Fm}^{-1}\right)$ is the dielectric permittivity, $d(\mathrm{~m})$ is the depth of the capacitor and $K^{\prime}$ and $K$ are the real and imaginary quarters of periods of the Jacobi elliptic function (3.5) and (3.6). Assuming $d=5(\mathrm{~mm})$ and $\varepsilon=\varepsilon_{0}$, one can verify in Table 3 the capacitance values obtained by (6.3) and the software Flux 2D.

\subsection{Calculation of the Capacitance of a Capacitor with Any Geometry}

Another application example of the proposed method is presented to determinate the capacitance of a capacitor with any polygonal geometry. To find the prevertices of a device with curved geometry, is necessary to transform the curved portions into approximated segments of straight lines. The smaller these approximated segments, the more accurate will be the result. Data from the simulation of this device is presented in Table 4 and illustrated in Figure 12. 

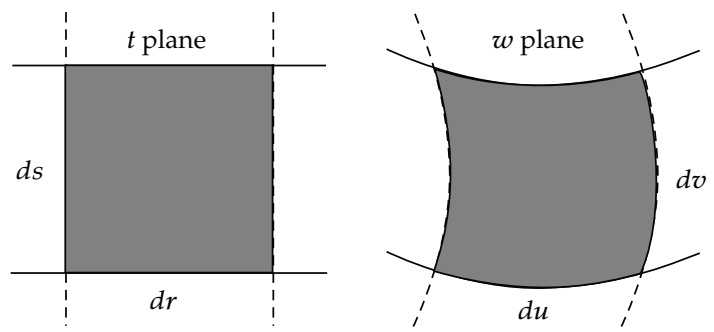

Figure 11: Curvilinear squares.

Table 3: Calculation of the capacitance.

\begin{tabular}{ll}
\hline $\begin{array}{l}\text { Curvilinear Squares Method } \\
\text { (Conformal Mapping) }\end{array}$ & $\begin{array}{l}\text { Finite Elements Method } \\
\text { (Software Flux 2D) }\end{array}$ \\
\hline $0.07663 p \mathrm{~F}$ & $0.07696 p \mathrm{~F}$ \\
\hline
\end{tabular}

In Figure 12, we define two lines of constant flux, given by $\overline{w_{10} w_{11}}$ Plate 1 and $\overline{w_{12} w_{13}}$ Plate 2, respectively. The two lines of constant flux are mapped into the complex planes $z$ and $t$, by means of the conformal mapping. The relations between the vertices and the prevertices are presented in Table 5.

With the rectangle in the complex $t$ plane, we calculate the device's capacitance using expression (6.3), where $K^{\prime}=t_{11}-t_{10}(\mathrm{~m})$ and $(2 \cdot K)=t_{11}-t_{12}(\mathrm{~m})$. Considering that $\varepsilon\left(\mathrm{Fm}^{-1}\right)$, $d(\mathrm{~m}), K^{\prime}$ and $K$ are the same as previously mentioned at Section 6.2.

Assuming $d=9(\mathrm{~mm})$ and $\varepsilon=\varepsilon_{0}$, in Table 6 are listed the capacitance values obtained by (6.3) and by the software Flux $2 D[20]$.

\subsection{Capacitance Calculation of an Eccentric Cylindrical Capacitor}

Figure 13 shows the cross-section of a cylindrical capacitor of length $d$, formed by two coaxial cylinders of radius $R_{1}$ and $R_{2}$. If $d \gg R_{1}$, so that we can neglect the distortion of the electric field that occurs at the ends of the cylinders. The external plate has a positive potential $V$, while the internal plate has a null potential.

Assuming that the plates are homogeneous along the length of the capacitor, and that $\psi=0$ is the eccentricity of the plates, the capacitance is defined for the concentric cylindrical capacitor as:

$$
C_{c}=2 \pi \varepsilon \frac{d}{\ln \left(R_{1} / R_{2}\right)} .
$$

Now, assuming a cylindrical capacitor of cross-section as shown in Figure 13, where the plates has the same radius, that is, $R_{1}=r_{1}$ and $R_{2}=r_{2}$, but with an eccentricity $\psi \neq 0$, as illustrated in Figure 14.

There is a certain difficulty to calculate the capacitance of a device with this geometry. However, a geometry can be find, where the capacitance will be easily calculated. Utilizing 
Table 4: Relationship between vertices, slopes, and prevertices.

\begin{tabular}{lcc}
\hline Polygon vertices (Figure 12) & Slopes $\alpha_{n}$ & Prevertices (Genetic Algorithm) \\
\hline$w_{1}=-0.44+7.96 i$ & $\alpha_{1}=-0.0939$ & $x_{1}=5.908024$ \\
$w_{2}=-1.28+7.71 i$ & $\alpha_{2}=-0.0939$ & $x_{2}=5.911571$ \\
$w_{3}=-2.00+7.22 i$ & $\alpha_{3}=-0.0939$ & $x_{3}=5.915387$ \\
$w_{4}=-2.57+6.54 i$ & $\alpha_{4}=-0.0939$ & $x_{4}=5.919682$ \\
$w_{5}=-2.91+5.72 i$ & $\alpha_{5}=-0.0939$ & $x_{5}=5.924861$ \\
$w_{6}=-2.99+4.85 i$ & $\alpha_{6}=-0.0939$ & $x_{6}=5.931535$ \\
$w_{7}=-2.82+3.99 i$ & $\alpha_{7}=-0.0939$ & $x_{7}=5.941163$ \\
$w_{8}=-2.40+3.21 i$ & $\alpha_{8}=-0.0233$ & $x_{8}=5.958801$ \\
$w_{9}=-1.07+1.11 i$ & $\alpha_{9}=0.6805$ & $x_{9}=6.519177$ \\
$w_{10}=-5.97+1.11 i$ & $\alpha_{10}=-0.5000$ & $x_{10}=6.866081$ \\
$w_{11}=-5.97+0.00 i$ & $\alpha_{11}=-0.5000$ & $x_{11}=6.866082$ \\
$w_{12}=6.00+0.00 i$ & $\alpha_{12}=-0.5000$ & $x_{12}=4.856315$ \\
$w_{13}=6.00+4.00 i$ & $\alpha_{13}=-0.6720$ & $x_{13}=4.856693$ \\
$w_{14}=0.99+0.99 i$ & $\alpha_{14}=0.8525$ & $x_{14}=5.166399$ \\
$w_{15}=2.40+3.20 i$ & $\alpha_{15}=-0.0233$ & $x_{15}=5.853812$ \\
$w_{16}=2.81+3.98 i$ & $\alpha_{16}=-0.0939$ & $x_{16}=5.871332$ \\
$w_{17}=2.99+4.84 i$ & $\alpha_{17}=-0.0939$ & $x_{17}=5.881014$ \\
$w_{18}=2.90+5.72 i$ & $\alpha_{18}=-0.0939$ & $x_{18}=5.887704$ \\
$w_{19}=2.57+6.54 i$ & $\alpha_{19}=-0.0939$ & $x_{19}=5.892895$ \\
$w_{20}=2.01+7.22 i$ & $\alpha_{20}=-0.0939$ & $x_{20}=5.897198$ \\
$w_{21}=1.28+7.71 i$ & $\alpha_{21}=-0.0939$ & $x_{21}=5.901012$ \\
$w_{22}=0.43+7.96 i$ & $\alpha_{22}=-0.0939$ & $x_{22}=5.904559$ \\
\hline & &
\end{tabular}

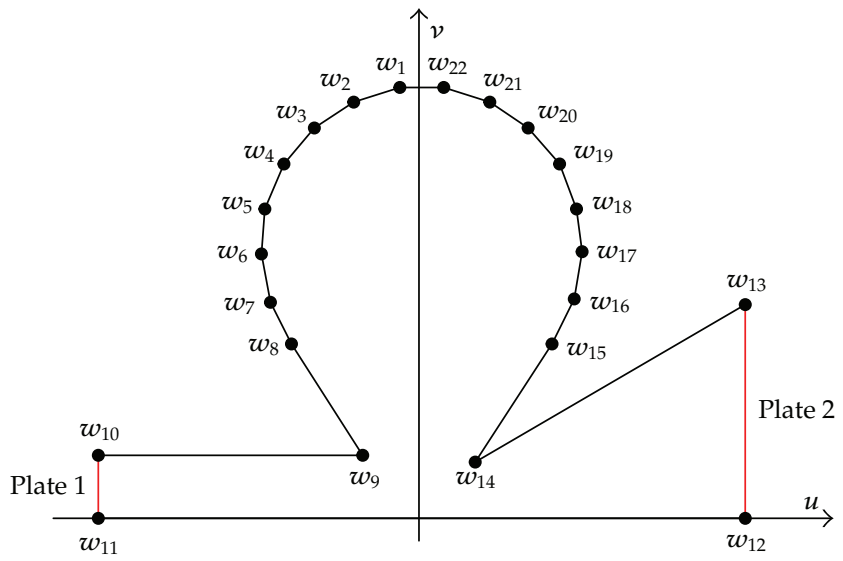

Figure 12: Device with any polygonal geometry.

Table 5: Relationship between the $w, z$, and $t$ planes for the device of any geometry.

\begin{tabular}{llc}
\hline$w$ plane & $z$ plane & $t$ plane \\
\hline$w_{10}=-5.97+1.11 i$ & $z_{10}=6.86608103$ & $t_{10}=7.90+0.000 i$ \\
$w_{11}=-5.97+0.00 i$ & $z_{11}=6.86608204$ & $t_{11}=7.90+1.568 i$ \\
$w_{12}=6.00+0.00 i$ & $z_{12}=4.85631578$ & $t_{12}=-7.90+1.568 i$ \\
$w_{13}=6.00+4.00 i$ & $z_{13}=4.85669351$ & $t_{13}=-7.90+0.000 i$ \\
\hline
\end{tabular}


Table 6: Calculation of the capacitance of a device with any polygonal geometry.

\begin{tabular}{ll}
\hline $\begin{array}{l}\text { Curvilinear Squares Method } \\
\text { Conformal Mapping }\end{array}$ & $\begin{array}{l}\text { Finite Elements Method } \\
\text { Software Flux 2D }\end{array}$ \\
\hline $7.9082 \mathrm{fF}$ & $7.9125 \mathrm{fF}$ \\
\hline
\end{tabular}

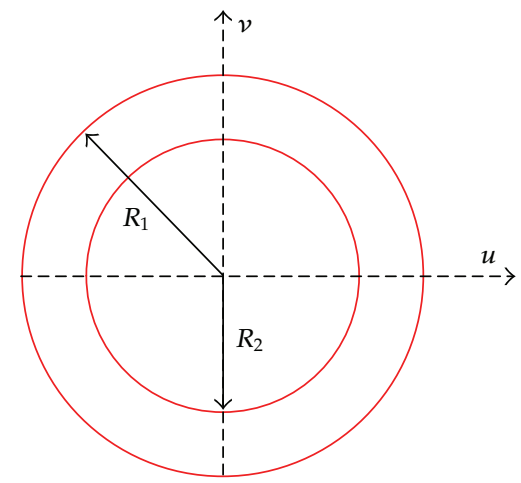

Figure 13: Concentric cylindrical capacitor.

algebraic manipulation, a conformal mapping can be developed transforming two eccentric circles into two concentric circles

$$
w(z)=t \cdot \frac{R_{1}}{r_{1}} \cdot e^{i \theta} \cdot \frac{d\left(z-z_{a}\right)-s\left(z_{b}-z_{a}\right)}{d\left(z-z_{a}\right)-t\left(z_{b}-z_{a}\right)}
$$

where $\theta, s$, and $t$ are real, $z_{a}$ are the points on the external plate, $z_{b}$ are the points on the internal plate, and $s$ and $t$ are the roots of the equation:

$$
\begin{gathered}
s \cdot t=r_{1}^{2}, \\
(d-s) \cdot(d-t)=r_{2}^{2},
\end{gathered}
$$

in (6.6), $d=\left|z_{a}-z_{b}\right|=\psi>0$ and $z_{a}, z_{b}, r_{1}, r_{2}, R_{1}, R_{2}>0$ and $z_{a} \neq z_{b}$, from this equation, it is possible to write

$$
\begin{aligned}
& \frac{r_{2}}{r_{1}} \cdot\left|\frac{t}{(d-t)}\right|=\frac{R_{2}}{R_{1}} \\
& -\frac{-d^{2}-r_{1}^{2}+r_{2}^{2}+\sqrt{-4 d^{4} r_{1}^{2}+\left(d^{2}+r_{1}^{2}-r_{2}^{2}\right)^{2}}}{2 d}=t \\
& -\frac{-d^{2}-r_{1}^{2}+r_{2}^{2}-\sqrt{-4 d^{4} r_{1}^{2}+\left(d^{2}+r_{1}^{2}-r_{2}^{2}\right)^{2}}}{2 d}=s .
\end{aligned}
$$

Therefore, assuming as data $R_{1}=r_{1}=10, R_{2}=r_{2}=5$, and $\psi=3$ for Figure 14, and using the conformal mapping (6.6), a new circular geometry at the complex $z$ plane is 


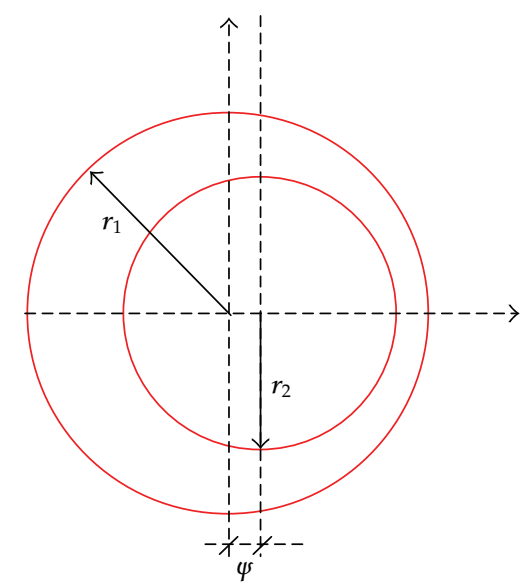

Figure 14: Eccentric cylindrical capacitor.

Table 7: Relation between the $w$ and $z$ planes for the eccentric cylindrical capacitor.

\begin{tabular}{lc}
\hline$w$ plane & $z$ plane \\
\hline$r_{1}=10.0000$ & $r_{1}^{\prime}=17.4788$ \\
$r_{2}=5.0000$ & $r_{2}^{\prime}=10.0000$ \\
$\psi=3$ & $\psi^{\prime}=0$ \\
\hline
\end{tabular}

obtained. In this new geometry, the circles are concentric and the relationship between $w$ plane and $z$ plane are listed in Table 7.

Thus, with the data of Table 7 in hands, and assuming $d=50$, the capacitance of an eccentric cylindrical capacitor is calculated, using (6.6) jointly with (6.4). In Table 8 are presented the capacitance values of an concentric device, and an eccentric device, whose values where given in the previous paragraph.

It is interesting to observe in Table 8 that the eccentricity value $\psi$ modifies the capacitance value, increasing it.

\subsection{Permeance of a Geometry Polygon Device of Straight Section}

The following presents an example that applies the method proposed here to estimate the permeance value of a polygonal geometry device of straight section. The simulation data of this device is presented in Table 9 and illustrates in Figure 15.

Figure 15, represents the complex $w$ plane, defining in this plane, two constant flux lines, given by $\overline{w_{5} w_{6}}$ (Plate 1$)$ and $\overline{w_{11} w_{12}}$ (Plate 2$)$, respectively. The two lines of constant flux are mapped into the complex $z$ and $t$ planes, by means of the conformal mapping. The relations between the vertices and the prevertices are presented at Table 10.

With the rectangle in the complex $t$ plane in hands, one can calculate the permeance of the device, using expression (6.8) depending on the real and imaginary quarters of periods of the Jacobi elliptic function (3.5) and (3.6)

$$
P=\mu_{0} \cdot \frac{p_{b} \cdot(2 \cdot K)}{K^{\prime}} .
$$


Table 8: Capacitances of the concentric and eccentric cylindrical capacitors.

\begin{tabular}{ll}
\hline $\begin{array}{l}\text { Concentric Capacitor } \\
\text { Figure 13 }\end{array}$ & $\begin{array}{l}\text { Eccentric Capacitor } \\
\text { Figure 14 }\end{array}$ \\
\hline $37.645 n \mathrm{~F}$ & $48.440 n \mathrm{~F}$ \\
\hline
\end{tabular}

Table 9: Relation between vertices, slopes, and prevertices.

\begin{tabular}{lcc}
\hline Polygon vertices (Figure 15) & Slopes $\alpha_{n}$ & Prevertices (Genetic Algorithm) \\
\hline$w_{1}=-1.99+3.00 i$ & $\alpha_{1}=0.5000$ & $x_{1}=1.000000$ \\
$w_{2}=-1.99+6.00 i$ & $\alpha_{2}=-0.5000$ & $x_{2}=1.768640$ \\
$w_{3}=-4.00+6.00 i$ & $\alpha_{3}=-0.5000$ & $x_{3}=1.853695$ \\
$w_{4}=-4.00+3.00 i$ & $\alpha_{4}=0.5000$ & $x_{4}=1.853696$ \\
$w_{5}=-6.00+3.00 i$ & $\alpha_{5}=-0.5000$ & $x_{5}=1.853777$ \\
$w_{6}=-6.00+1.90 i$ & $\alpha_{6}=-0.5000$ & $x_{6}=1.854082$ \\
$w_{7}=-1.00+1.90 i$ & $\alpha_{7}=0.6476$ & $x_{7}=1.854101$ \\
$w_{8}=-2.00+0.00 i$ & $\alpha_{8}=-0.6476$ & $x_{8}=1.855555$ \\
$w_{9}=2.00+0.00 i$ & $\alpha_{9}=-0.6476$ & $x_{9}=2.141762$ \\
$w_{10}=1.00+1.90 i$ & $\alpha_{10}=0.6476$ & $x_{10}=2.143211$ \\
$w_{11}=6.00+1.90 i$ & $\alpha_{11}=-0.5000$ & $x_{11}=2.143230$ \\
$w_{12}=6.00+3.00 i$ & $\alpha_{12}=-0.5000$ & $x_{12}=2.143535$ \\
$w_{13}=4.00+3.00 i$ & $\alpha_{13}=0.5000$ & $x_{13}=2.143616$ \\
$w_{14}=4.00+6.00 i$ & $\alpha_{14}=-0.5000$ & $x_{14}=2.143617$ \\
$w_{15}=1.99+6.00 i$ & $\alpha_{15}=-0.5000$ & $x_{15}=2.228698$ \\
$w_{16}=1.99+3.00 i$ & $\alpha_{16}=0.5000$ & $x_{16}=2.997312$ \\
\hline
\end{tabular}

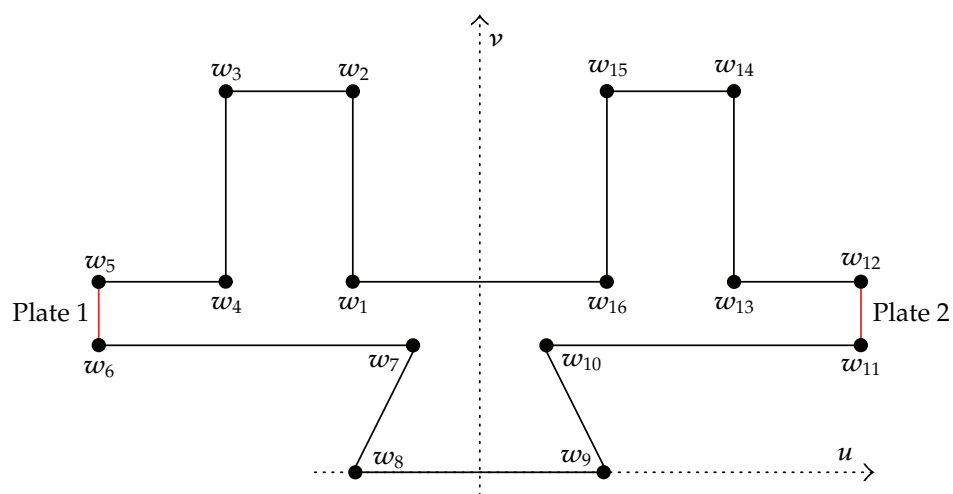

Figure 15: Geometry polygon device of straight section.

Table 10: Relation between the $w, z$, and $t$ planes for a given device with polygonal geometry.

\begin{tabular}{lcc}
\hline$w$ plane & $z$ plane & $t$ plane \\
\hline$w_{5}=-6.00+3.00 i$ & $z_{5}=1.853695$ & $t_{5}=-6.981+1.57 i$ \\
$w_{6}=-6.00+1.90 i$ & $z_{6}=1.853696$ & $t_{6}=-6.981+0.00 i$ \\
$w_{11}=6.00+1.90 i$ & $z_{11}=2.143616$ & $t_{11}=6.981+0.00 i$ \\
$w_{12}=6.00+3.00 i$ & $z_{12}=2.143617$ & $t_{12}=6.981+1.57 i$ \\
\hline
\end{tabular}


In which $(2 \cdot K)=t_{11}-t_{6}(\mathrm{~m}), K^{\prime}=t_{12}-t_{11}(\mathrm{~m})$ and $p_{b}(\mathrm{~m})$ is the depth of the device, in this case, $p_{b}=1$. The permeance value found for the device shown at Figure 15, using the proposed methodology in (6.8), is $11.17 \mathrm{nH}$.

\section{Conclusion}

The results presented in Sections 6.1, 6.2, 6.3, 6.4, and 6.5 showed that the solution of electromagnetic problems related to geometry, can be simplified, using the conformal mapping. Also, the Schwarz-Christoffel Transformation appears to be a suitable and effective tool to solve problems involving complicated geometries forms, considering the conformal mapping property of modifying only the geometry of a polygonal structure, preserving the physical magnitude corresponding to each point at the plane. Regarding the mathematical operator developed to help the optimization method, there was a reduction of approximately $38 \%$ on average in the number of generations $g$ to be achieved, when compared with the genetic algorithm without the operator. Therefore, the guided evolution operator, promoted a stimulus in the used optimization method, reducing the time spent in the search of optimized parameters. In Sections 6.2 and 6.4, the capacitance calculation performed by the proposed method was compared with results obtained by Flux $2 D$ software, validating the proposed method. The computational processes were reduced comparing with sophisticated softwares used to produce electromagnetic fields, leading an improvement in the calculation performance of some devices with odd geometry. As a suggestion for future studies, the methodology using conformal mapping allied to the developed mathematical operator, could be applied in the design of optimized electric devices, of any desired geometry. The calculations presented here are useful as a theoretical framework to guide experiments on capacitors from different geometry and introduce a new concept in the permeance estimation of electrical devices.

\section{Acknowledgments}

The authors acknowledge the Capes Foundation, Ministry of Education of Brazil, for the financial support scholarship Proc. no. BEX 3873/10-2. This work was supported in part by the brazilian agencies Coordenação de Aperfeiçoamento de Pessoal de Nível Superior (CAPES/MEC) and Conselho Nacional de Desenvolvimento Científico e Tecnológico (CNPq) under Grant 479186/2006-5/Universal.

\section{References}

[1] P. Henrici, Applied and Computational Complex Analysis, vol. 3 of Pure and Applied Mathematics, John Wiley \& Sons, New York, NY, USA, 1986.

[2] M. R. Spiegel, Complex Variable, McGraw-Hill, New York, NY, USA, 1967.

[3] J. D. Kraus and K. R. Carver, Electromagnetics, McGraw-Hill, New York, NY, USA, 1973.

[4] W. P. Calixto, E. G. Marra, L. C. Brito, and B. P. Alvarenga, "A new methodology to calculate carter factor using geneticalgorithms," International Journal of Numerical Modelling: Electronic Networks, Devices and Fields. In press.

[5] Z. Nehari, Conformal Mapping, McGraw-Hill, New York, NY, USA, 1952.

[6] H. Cohn, Conformal Mapping on Riemann Surfaces, Dover, New York, NY, USA, 1967.

[7] W. J. Gibbs, Conformal Transformations in Electrical Engineering, Chapman and Hall, London, UK, 1958.

[8] G. C. Wen, Conformal Mappings and Boundary Value Problems, vol. 106 of Translations of Mathematical Monographs, American Mathematical Society, Providence, RI, USA, 1992. 
[9] L. N. Trefethen, "Numerical computation of the Schwarz-Christoffel transformation," Society for Industrial and Applied Mathematics, vol. 1, no. 1, pp. 82-102, 1980.

[10] T. A. Driscoll and S. A. Vavasis, "Numerical conformal mapping using cross-ratios and Delaunay triangulation," SIAM Journal on Scientific Computing, vol. 19, no. 6, pp. 1783-1803, 1998.

[11] E. Costamagna, "On the numerical inversion of the schwarz-christoffel conformal transformation," IEEE Transactions on Microwave Theory and Techniques, vol. 35, no. 1, pp. 35-40, 1987.

[12] T. A. Driscoll and L. N. Trefethen, Schwarz-Christoffel Mapping, vol. 8 of Cambridge Monographs on Applied and Computational Mathematics, Cambridge University Press, Cambridge, UK, 2002.

[13] M. Abramowitz and I. A. Stegun, Handbook of Mathematical Functions: with Formulas, Graphs, and Mathematical Tables, Dover, New York, NY, USA, 1968.

[14] S. C. Milne, Infinite Families of Exact Sums of Squares Formulas, Jacobi Elliptic Functions, Continued Fractions, and Schur Functions, vol. 5 of Developments in Mathematics, Kluwer Academic Publishers, Boston, Mass, USA, 2002.

[15] W. P. Calixto, Application of conformal mapping to the calculus of Carter's factor, M.S. thesis, Electrical \& Computer Engineering School, Federal University of Goias, Goiania, Brazil, 2008.

[16] Z. Michalewicz, Genetic Algorithms + Data Structures = Evolution Programs, Artificial Intelligence, Springer, Berlin, Germany, 1992.

[17] Z. Michalewicz and D. B. Fogel, How to Solve it: Modern Heuristics, Springer, Berlin, Germany, 2000.

[18] J. H. Holland, Adaptation in Natural and Artificial Systems: an Introductory Analysis with Applications to Biology, Control, and Artificial Intelligence, University of Michigan Press, Ann Arbor, Mich, USA, 1975.

[19] F. Herrera, M. Lozano, and J. L. Verdegay, "Crossover operators and offspring selection for real coded genetic algorithms," Tech. Rep., Department of Intelligence of the Computation and Artificial intelligence, University of Granada, Granada, Spain, 1994.

[20] Cedrat, Flux 2D User's Guide, Cedrat, Grenoble, France, 2000.

[21] R. E. Collins, Mathematical Methods for Physicists and Engineers, Dover, New York, NY, USA, 2nd edition, 1999. 


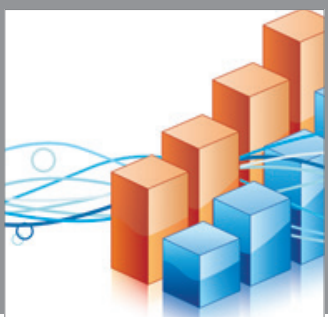

Advances in

Operations Research

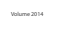

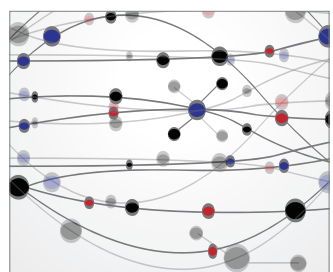

\section{The Scientific} World Journal
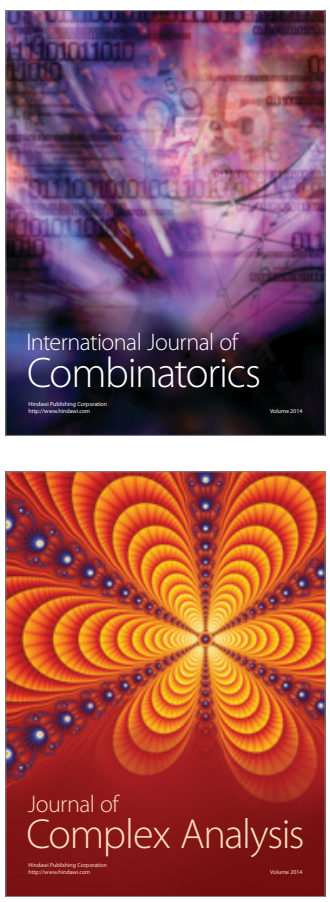

International Journal of

Mathematics and

Mathematical

Sciences
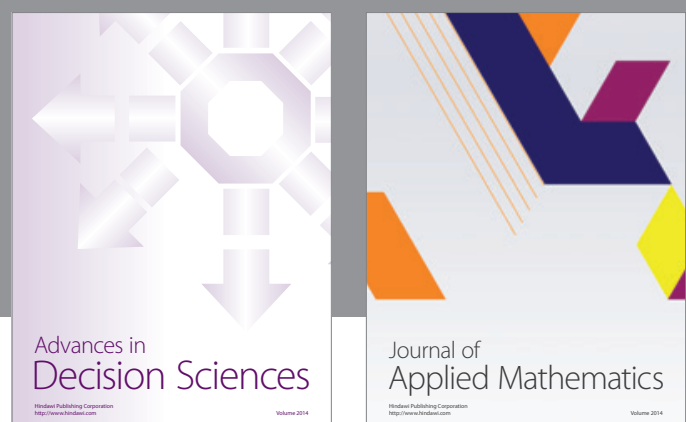

Journal of

Applied Mathematics
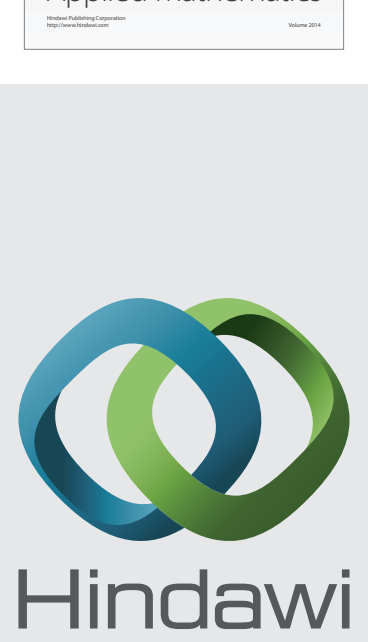

Submit your manuscripts at http://www.hindawi.com
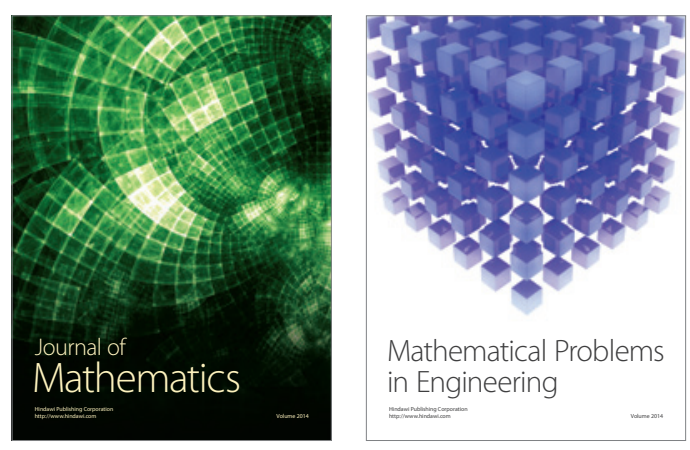

Mathematical Problems in Engineering
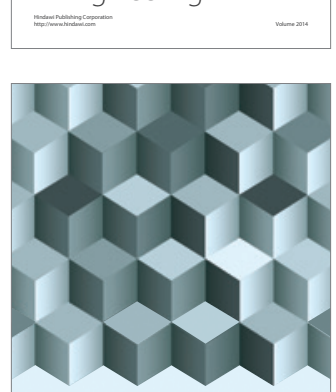

Journal of

Function Spaces
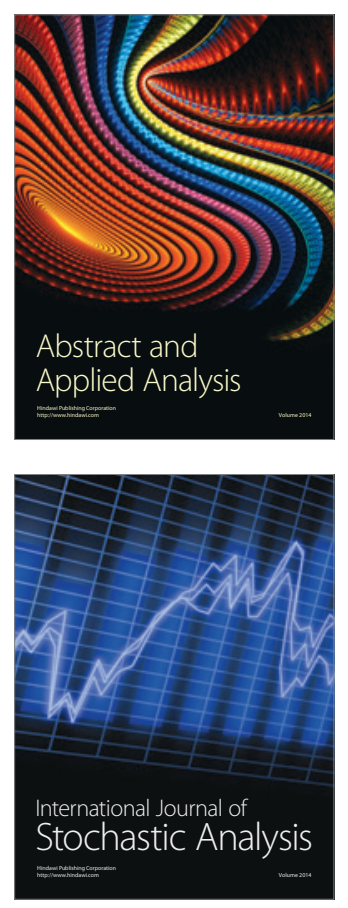

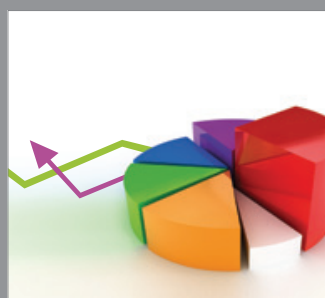

ournal of

Probability and Statistics

Promensencen
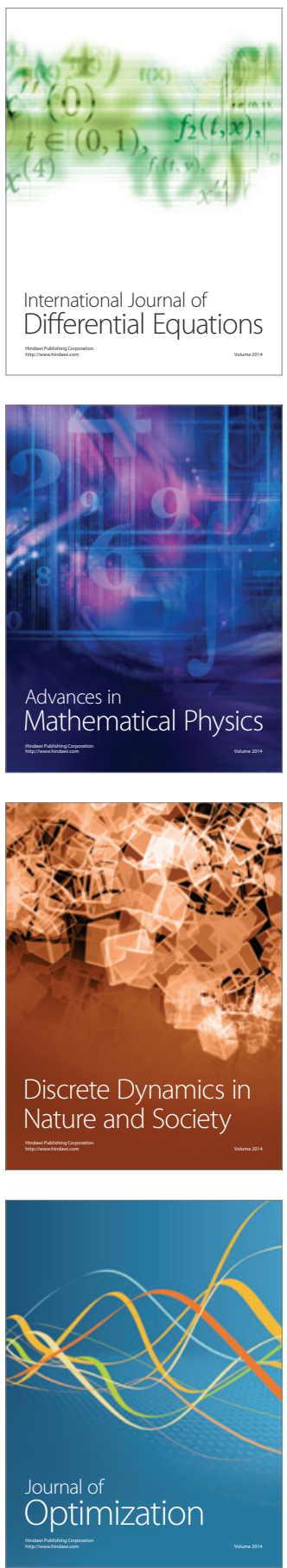